JOURNAL OF

FUNCTION SPACES AND APPLICATIONS

Volume 5, Number 2 (2007), 133-150
(C) 2007, Scientific Horizon http://www.jfsa.net

\title{
Bounds on the effective behavior of a homogenized generalized Reynolds equation
}

\author{
Dag Lukkassen, Annette Meidell and Peter Wall
}

(Communicated by Björn Birnir )

2000 Mathematics Subject Classification. 35B27, 74Q20.

Keywords and phrases. Homogenization, effective properties, bounds, Reynolds equation.

Abstract. We study upper and lower bounds for estimating the effective behavior described by homogenizing a problem which is a generalization of the Reynold equation. All cases when these bounds coincide are also found.

\section{Introduction}

We consider an equation of the form

(1)

$\frac{\partial}{\partial x_{1}}\left(a_{1}\left(x, \frac{x}{\varepsilon}\right) \frac{\partial u_{\varepsilon}}{\partial x_{1}}-b_{1}\left(x, \frac{x}{\varepsilon}\right)\right)+\frac{\partial}{\partial x_{2}}\left(a_{2}\left(x, \frac{x}{\varepsilon}\right) \frac{\partial u_{\varepsilon}}{\partial x_{2}}-b_{2}\left(x, \frac{x}{\varepsilon}\right)\right)=f(x)$,

$x \in \Omega \subset R^{2}, u_{\varepsilon} \in H_{0}^{1}(\Omega)$. Here, $a_{i}$ and $b_{i}$ are assumed to be piecewise continuous in the first variable and measurable and periodic relative to a cell $Y=[0,1]^{2}$ in the second variable. In addition, we assume that there exist constants $k_{-}$and $k_{+}$such that

$$
0<k_{-} \leq a_{i}(x, y) \leq k_{+}<\infty \text { and }\left|b_{i}(x, y)\right| \leq k_{+}
$$


for all $x$ and $y$. Moreover, $\varepsilon>0$ is a small parameter.

Many important physical problems can be described by a partial differential equation of the type (1). The standard example is stationary heat conduction in which $b_{i}=0$. An other example concerns flow behavior between two surfaces in relative motion in the theory of lubrication for thin films for which $f(x)=0$ and $c_{i} b_{i}^{3}(x, y)=a_{i}(x, y)=a(x, y)$ for some constant $c_{i}$ which is propositional to the relative motion. For that special case (1) is the incompressible Reynolds equation.

For simplicity we put $f=0$. The corresponding weak formulation takes the form: Find $u_{\varepsilon} \in H_{0}^{1}(\Omega)$ such that

$$
\int_{\Omega}\left(a_{1}\left(x, \frac{x}{\varepsilon}\right) \frac{\partial u_{\varepsilon}}{\partial x_{1}}-b_{1}\left(x, \frac{x}{\varepsilon}\right)\right) \frac{\partial v}{\partial x_{1}}+\left(a_{2}\left(x, \frac{x}{\varepsilon}\right) \frac{\partial u_{\varepsilon}}{\partial x_{2}}-b_{2}\left(x, \frac{x}{\varepsilon}\right)\right) \frac{\partial v}{\partial x_{2}} d x=0
$$

for all $v \in H_{0}^{1}(\Omega)$. The equivalent variational formulation is then: Find $u_{\varepsilon} \in H_{0}^{1}(\Omega)$ such that

$$
F_{\varepsilon}\left(D u_{\varepsilon}\right)=\min _{v \in H_{0}^{1}(\Omega)} F_{\varepsilon}(D v)
$$

where

$$
F_{\varepsilon}(D v)=\int_{\Omega} f_{\varepsilon}(x, D v) d x
$$

and

$$
f_{\varepsilon}(x, \xi)=f\left(x, \frac{x}{\varepsilon}, \xi\right)=\sum_{i=1}^{2} \frac{1}{2} a_{i}\left(x, \frac{x}{\varepsilon}\right) \xi_{i}^{2}-b_{i}\left(x, \frac{x}{\varepsilon}\right) \xi_{i} .
$$

Putting $v=u_{\varepsilon}$ into the weak formulation (2) we observe that the "energy" $F_{\varepsilon}\left(D u_{\varepsilon}\right)$ is given by

$$
F_{\varepsilon}\left(D u_{\varepsilon}\right)=-\frac{1}{2} \int_{\Omega} b_{1}\left(x, \frac{x}{\varepsilon}\right) \frac{\partial u_{\varepsilon}}{\partial x_{1}}+b_{2}\left(x, \frac{x}{\varepsilon}\right) \frac{\partial u_{\varepsilon}}{\partial x_{2}} d x .
$$

The right hand side (multiplied with some known constant) often represent some important physical property. Examples of such properties are resultant force, resultant moment, total heat flux, total current, etc. Consequently, in many cases the calculation of $F_{\varepsilon}\left(u_{\varepsilon}\right)$ is the main purpose of the investigation.

Using the theory of gamma-convergence, it is possible to prove that

$$
F_{\varepsilon}\left(D u_{\varepsilon}\right) \rightarrow F_{0}(D \bar{u})=\min _{u \in H_{0}^{1}(\Omega)} F_{0}(D u),
$$

where

$$
F_{0}(D u)=\int_{\Omega} f_{0}(x, D u) d x
$$




$$
f_{0}(x, \xi)=\min _{u \in W_{p e r}(Y)} \int_{Y} f(x, y, D u+\xi) d y
$$

and $W_{\text {per }}(Y)$ is the space of $Y$-periodic functions belonging to the Sobolev space $H^{1}(Y)$. This result can also be proved by using so-called two-scale convergence (see [12]). Putting

$$
A=\left[\begin{array}{cc}
a_{1} & 0 \\
0 & a_{2}
\end{array}\right], \quad B=\left[\begin{array}{l}
b_{1} \\
b_{2}
\end{array}\right]
$$

we have that $f$ and $f_{0}$ can be written on the forms

$$
\begin{aligned}
& f(x, y, \xi)=\frac{1}{2} \xi \cdot A(x, y) \xi-B(x, y) \cdot \xi \\
& f_{0}(x, \xi)=\frac{1}{2} \xi \cdot A_{0} \xi-B_{0} \cdot \xi+f_{0}(x, 0) .
\end{aligned}
$$

Here,

$$
\begin{aligned}
A_{0} \xi & =\int_{Y} A\left(D u_{\xi}+\xi\right) d y \\
B_{0} & =\int_{Y} B-A D u_{0} d y
\end{aligned}
$$

where $u_{\xi} \in W_{\text {per }}(Y)$ and $u_{0} \in W_{\text {per }}(Y)$ are the solutions of the local problems

$$
\begin{aligned}
& \int_{Y} D v \cdot A\left(D u_{\xi}+\xi\right) d y=0, \quad \forall v \in W_{p e r}(Y), \\
& \int_{Y} D v \cdot\left(A D u_{0}-B\right) d y=0, \quad \forall v \in W_{p e r}(Y) .
\end{aligned}
$$

For the latter problem we note that the corresponding variational problem takes the form

$$
f_{0}(x, 0)=\min _{v \in W_{p e r}(Y)} \int_{Y} \frac{1}{2} D v \cdot A D v-B \cdot D v d y .
$$

Thus, by putting $v=u_{0}$ in (5), we get that

$$
f_{0}(x, 0)=\int_{Y} \frac{1}{2} D u_{0} \cdot A D u_{0}-B \cdot D u_{0} d y=-\frac{1}{2} \int_{Y} B \cdot D u_{0} d y .
$$

In this paper we find sharp upper and lower bounds $f^{-}(x, \xi)$ and $f^{+}(x, \xi)$ for $f_{0}(x, \xi)$. These bounds are described by integral averages in orthogonal directions and can easily be found explicitly or at least by performing 
numerical integration. All cases when these bounds coincide are also found. When these bounds are close to each other, we are able to find close upper and lower bounds $F_{-}\left(D u_{-}\right)$and $F_{+}\left(D u_{+}\right)$for the homogenized energy $F_{0}(D \bar{u})$ by solving the global problems

$$
F_{-}\left(D u_{-}\right)=\min _{u \in W_{0}(\Omega)} F_{+}(D u) \text { and } F_{+}\left(D u_{+}\right)=\min _{u \in W_{0}(\Omega)} F_{+}(D u),
$$

where

$$
F_{ \pm}(D u)=\int_{\Omega} f^{ \pm}(x, D u) d x .
$$

The bounds are closely connected to the ones obtained in [5] (see also [6], [7], [8] and [9]). The derivation is however different due to the presence of $b_{i}(x, y)$.

\section{The main result}

For $(i, j)=(1,2)$ or $(i, j)=(2,1)$ let us define the following constants associated with the functions $a_{i}=a_{i}\left(x,\left(y_{1}, y_{2}\right)\right)$ and $b_{i}=b_{i}\left(x,\left(y_{1}, y_{2}\right)\right)$.

$$
\begin{aligned}
a_{i}^{+}(x)= & \left(\int_{0}^{1}\left(\int_{0}^{1} a_{i} d y_{j}\right)^{-1} d y_{i}\right)^{-1}, \\
a_{i}^{-}(x)= & \int_{0}^{1}\left(\int_{0}^{1} a_{i}^{-1} d y_{i}\right)^{-1} d y_{j}, \\
c_{i}^{+}(x)= & \left(\int_{0}^{1}\left(\int_{0}^{1} a_{i} d y_{j}\right)^{-1} d y_{i}\right)^{-1} \int_{0}^{1} \frac{\int_{0}^{1} b_{i} d y_{j}}{\int_{0}^{1} a_{i} d y_{j}} d y_{i}, \\
c_{i}^{-}(x)= & \int_{0}^{1}\left(\int_{0}^{1} a_{i}^{-1} d y_{i}\right)^{-1} \int_{0}^{1} \frac{b_{i}}{a_{i}} d y_{i} d y_{j}, \\
d_{i}^{+}(x)= & \frac{1}{2}\left(\int_{0}^{1}\left(\int_{0}^{1} a_{i} d y_{j}\right)^{-1} d y_{i}\right)^{-1}\left(\int_{0}^{1} \frac{\int_{0}^{1} b_{i} d y_{j}}{\int_{0}^{1} a_{i} d y_{j}} d y_{i}\right)^{2} \\
& -\frac{1}{2} \int_{0}^{1} \frac{\left(\int_{0}^{1} b_{i} d y_{j}\right)^{2}}{\int_{0}^{1} a_{i} d y_{j}} d y_{i}, \\
& -\frac{1}{2} \int_{0}^{1} \int_{0}^{1} \frac{\int_{0}^{1} b_{i}^{2}}{a_{i}} d y_{i} d y_{j} .
\end{aligned}
$$

The main result of this paper reads: 
Theorem 1. It holds that

$$
f^{-}(x, \xi) \leq f_{0}(x, \xi) \leq f^{+}(x, \xi)
$$

where

$$
\begin{aligned}
& f^{-}(x, \xi)=\sum_{i=1}^{2}\left(\frac{1}{2} a_{i}^{-}(x) \xi_{i}^{2}-c_{i}^{-}(x) \xi_{i}+d_{i}^{-}(x)\right) \\
& f^{+}(x, \xi)=\sum_{i=1}^{2}\left(\frac{1}{2} a_{i}^{+}(x) \xi_{i}^{2}-c_{i}^{+}(x) \xi_{i}+d_{i}^{+}(x)\right) .
\end{aligned}
$$

Moreover, $f^{-}=f^{+}$if and only if $a_{i}=a_{i}^{i} a_{i}^{j}$ and $b_{i}=a_{i}^{j} f_{i}^{i}+f_{i}^{j}(j \neq i)$ where $a_{i}^{k}$ and $f_{i}^{k}$ are functions of the form

$$
a_{i}^{k}=a_{i}^{k}\left(x, y_{k}\right), f_{i}^{k}=f_{i}^{k}\left(x, y_{k}\right) .
$$

Remark 1. In the special case when $b_{i}=0$ the bounds in Theorem 1 reduces to the ones obtained in [7] concerning the homogenized $p$-Laplace equation for the case $p=2$.

Remark 2. As a consequence of Theorem 1 we obtain the inequality $f^{-} \leq f^{+}$. In particular this gives the inequality $a_{i}^{-}(x) \leq a_{i}^{+}(x)$, which has been known in the theory of inequalities for quite a long time (see e.g. [3, p. 148], [1] or [2, p. 170]).

Remark 3. The above theorem shows that $f^{+}(x, \xi)=f_{0}(x, \xi)=$ $f^{-}(x, \xi)$ if $a_{i}=a_{i}^{i} a_{i}^{j}$ and $b_{i}=a_{i}^{j} f_{i}^{i}+f_{i}^{j}$. For this case

$$
\begin{aligned}
a_{i}^{-}(x)= & a_{i}^{+}(x) \\
= & \left(\int_{0}^{1} a_{i}^{j} d y_{j}\right)\left(\int_{0}^{1}\left(a_{i}^{i}\right)^{-1} d y_{i}\right)^{-1}, \\
c_{i}^{-}(x)= & c_{i}^{+}(x) \\
= & \left(\int_{0}^{1} a_{i}^{j} d y_{j}\right)\left(\int_{0}^{1}\left(a_{i}^{i}\right)^{-1} d y_{i}\right)^{-1} \\
& \times\left(\int_{0}^{1} \frac{f_{i}^{i}}{a_{i}^{i}} d y_{i}+\frac{\int_{0}^{1} f_{i}^{j} d y_{j}}{\int_{0}^{1} a_{i}^{j} d y_{j}} \int_{0}^{1}\left(a_{i}^{i}\right)^{-1} d y_{i}\right), \\
d^{-}(x)= & d^{+}(x) \\
= & \frac{1}{2}\left(\int_{0}^{1} a_{i}^{j} d y_{j}\right)\left(\left(\int_{0}^{1}\left(a_{i}^{i}\right)^{-1} d y_{i}\right)^{-1}\right. \\
& \left.\times\left(\int_{0}^{1} \frac{f_{i}^{i}}{a_{i}^{i}} d y_{i}\right)^{2}-\int_{0}^{1} \frac{\left(f_{i}^{i}\right)^{2}}{a_{i}^{i}} d y_{i}\right) .
\end{aligned}
$$


This is seen directly for $a_{i}^{ \pm}(x)$ and $b_{i}^{ \pm}(x)$. The expression for $d_{i}^{ \pm}(x)$ is however more easily verified by computing the values of (14) and (15) below, and next adding these terms.

Example 1. As an example we consider the case when the unit cell $Y$ consists of two materials, material 1 and material 2 with conductivities $a_{1}(x, y)=a_{2}(x, y)=1$ and $a_{1}(x, y)=a_{2}(x, y)=2$, respectively. Material 2 occupies a square with size $l \times l$ and material 2 is the surrounding material. In both materials we assume that $b_{1}(x, y)=b_{2}(x, y)=0$. Due to symmetry, it holds that $f_{0}(x, \xi)=a\left(\xi_{1}^{2}+\xi_{2}^{2}\right)$. In the table below we have listed $a$ and $a^{ \pm}=a_{1}^{ \pm}=a_{2}^{ \pm}$for the case $l=0.5$ and $l=0.9$.

\begin{tabular}{|c|c|c|c|}
\hline \hline$l$ & $a$ & $a^{+}$ & $a^{-}$ \\
\hline 0.5 & 1.6903 & 1.7143 & 1.6667 \\
\hline 0.9 & 1.1494 & 1.1518 & 1.1474 \\
\hline \hline
\end{tabular}

The values in the second column are found numerically by using the Finite Element Method. The errors in these computations are estimated to be less than $4 \times 10^{-5}$.

For more information of numerical computation of effective properties and comparison with similar bounds we refer to the literature, see e.g. the thesis [10] and the article [11].

\section{Proof of the main result}

Let $\xi \in R^{2}$ and let $a_{i}$ and $b_{i}$ be measurable functions such that

$$
0<k_{-} \leq a_{i}(x) \leq k_{+}<\infty \text { and }\left|b_{i}(x)\right| \leq k_{+},
$$

and let $\mathcal{V}$ be a closed subspace of $\mathbf{L}^{2}(Y)$ of functions with zero mean value. Moreover, let $p_{\xi}=\left(p_{\xi 1}, p_{\xi 2}\right) \in \mathcal{V}$ be the solution of the problem

$$
\int_{Y} \sum_{i=1}^{2}\left(a_{i}\left(p_{\xi i}+\xi_{i}\right)+b_{i}\right) v_{i} d y=0, \text { for all } v=\left(v_{1}, v_{2}\right) \in \mathcal{V}
$$

We note that $p_{\xi}=p_{0}+\xi_{1} q_{1}+\xi_{2} q_{2}$, where $p_{0}=\left(p_{01}, p_{02}\right), q_{1}=\left(q_{11}, q_{12}\right)$ and $q_{2}=\left(q_{21}, q_{22}\right)$ are the solutions to the problems

$$
\int_{Y} \sum_{i=1}^{2}\left(a_{i} p_{0 i}+b_{i}\right) v_{i} d y=0, \text { for all } v=\left(v_{1}, v_{2}\right) \in \mathcal{V},
$$


$\int_{Y}\left(a_{1}\left(q_{11}+1\right) v_{1} d y+\int_{Y}\left(a_{2}\left(q_{12}+0\right) v_{2} d y=0\right.\right.$, for all $v=\left(v_{1}, v_{2}\right) \in \mathcal{V}$ $\int_{Y}\left(a_{1}\left(q_{21}+0\right) v_{1} d y+\int_{Y}\left(a_{2}\left(q_{22}+0\right) v_{2} d y=0\right.\right.$, for all $v=\left(v_{1}, v_{2}\right) \in \mathcal{V}$

respectively. The existence and uniqueness of the solution $p_{\xi}$ follows directly by Lax-Milgrams Lemma, since the above problem can be written on the form $A\left(p_{\xi}, v\right)=f(v)$, where

$$
\begin{aligned}
A\left(p_{\xi i}, v\right) & =\int_{Y} \sum_{i=1}^{2}\left(a_{i}\left(p_{\xi i}\right) v_{i} d y\right. \\
f(v) & =-\int_{Y} \sum_{i=1}^{2}\left(a_{i} \xi_{i}+b_{i}\right) v_{i} d y .
\end{aligned}
$$

By standard results, the solution $p_{\xi}$ is also the minimum-point of the functional $v \mapsto(1 / 2) A(v, v)-f(v)$, and, hence, also the minimum-point of the functional $v \mapsto \int_{Y} g(y, v+\xi) d y$, where

$$
g(y, \xi)=\sum_{i=1}^{2} \frac{1}{2} a_{i} \xi_{i}^{2}+b_{i} \xi_{i}
$$

(since its value is the same within an additive constant). Thus,

$$
g^{*}(\xi):=\min _{v \in \mathcal{V}} \int_{Y} g(y, v+\xi) d y=\int_{Y} g\left(y, p_{\xi}+\xi\right) d y
$$

Moreover, letting $g_{\xi_{i}}^{*}$ and $g_{\xi_{i}}$ denote the sub-differential

$$
\begin{aligned}
g_{\xi_{i}}^{*}(\xi) & =\frac{\partial g^{*}(\xi)}{\partial \xi_{i}} \\
g_{\xi_{i}}(y, \xi) & =\frac{\partial g(y, \xi)}{\partial \xi_{i}}
\end{aligned}
$$

we obtain that

$$
\begin{aligned}
g_{\xi_{i}}^{*}(\xi) & =\int_{Y} \sum_{j=1}^{2} g_{\xi_{j}}\left(y, p_{\xi}+\xi\right) \frac{\partial\left(p_{\xi}+\xi\right)_{j}}{\partial \xi_{i}} d y \\
& =\sum_{j=1}^{2} \int_{Y} g_{\xi_{j}}\left(y, p_{\xi}+\xi\right)\left(\frac{\partial p_{\xi j}}{\partial \xi_{i}}+\delta_{i j}\right) d y
\end{aligned}
$$




$$
\begin{aligned}
& =\sum_{j=1}^{2} \int_{Y} g_{\xi_{j}}\left(y, p_{\xi}+\xi\right)\left(q_{i j}+\delta_{i j}\right) d y \\
& =\int_{Y} g_{\xi_{i}}\left(y, p_{\xi}+\xi\right) d y+\sum_{j=1}^{2} \int_{Y} g_{\xi_{j}}\left(y, p_{\xi}+\xi\right)\left(q_{i j}\right) d y \\
& =\int_{Y} g_{\xi_{i}}\left(y, p_{\xi}+\xi\right) d y+\underbrace{\int_{Y} \sum_{j=1}^{2}\left(a_{j}\left(p_{\xi j}+\xi_{j}\right)+b_{j}\right) q_{i j} d y}_{=0 \text { by }(6)} \\
& =\int_{Y} g_{\xi_{i}}\left(y, p_{\xi}+\xi\right) d y,
\end{aligned}
$$

i.e.

$$
g_{\xi_{i}}^{*}(\xi)=\int_{Y} g_{\xi_{i}}\left(y, p_{\xi}+\xi\right) d y
$$

We are going to prove that

$$
f^{+}(x, \xi)=\min _{u \in V} \int_{Y} f(x, y, D u+\xi) d y
$$

where

$$
V=\left\{\varphi: \varphi \in W_{p e r}(Y), \varphi(y)=\varphi_{1}\left(y_{1}\right)+\varphi_{2}\left(y_{2}\right)\right\}
$$

This will directly show that $f^{+}(x, \xi)$ is an upper bound to $f_{0}(x, \xi)$. The corresponding Euler equation to (9) is: Find $u(y)=u_{1}\left(y_{1}\right)+u_{2}\left(y_{2}\right) \in V$ such that

$$
\begin{aligned}
& \int_{0}^{1} \int_{0}^{1}\left(a_{1}\left(x,\left(y_{1}, y_{2}\right)\right)\left(u_{1}^{\prime}\left(y_{1}\right)+\xi_{1}\right)-b_{1}\left(x,\left(y_{1}, y_{2}\right)\right)\right) v_{1}^{\prime}\left(y_{1}\right) d y_{2} d y_{1} \\
& \quad+\int_{0}^{1} \int_{0}^{1}\left(a_{2}\left(x,\left(y_{1}, y_{2}\right)\right)\left(u_{2}^{\prime}\left(y_{2}\right)+\xi_{2}\right)-b_{2}\left(x,\left(y_{1}, y_{2}\right)\right)\right) v_{2}^{\prime}\left(y_{2}\right) d y_{1} d y_{2}=0
\end{aligned}
$$

for all $v(y)=v_{1}\left(y_{1}\right)+v_{2}\left(y_{2}\right) \in V$, i.e.

$$
\begin{aligned}
& \int_{0}^{1}\left(\widetilde{a_{1}}\left(y_{1}\right)\left(u_{1}^{\prime}\left(y_{1}\right)+\xi_{1}\right)-\widetilde{b_{1}}\left(y_{1}\right)\right) v_{1}^{\prime}\left(y_{1}\right) d y_{1} \\
& \quad+\int_{0}^{1}\left(\widetilde{a_{2}}\left(y_{2}\right)\left(u_{2}^{\prime}\left(y_{2}\right)+\xi_{2}\right)-\widetilde{b_{2}}\left(y_{2}\right)\right) v_{2}^{\prime}\left(y_{2}\right) d y_{2}=0
\end{aligned}
$$


where

$$
\begin{aligned}
& \widetilde{a}_{i}\left(y_{i}\right)=\int_{0}^{1} a_{i}\left(x,\left(y_{1}, y_{2}\right)\right) d y_{j}, \quad i \neq j, \\
& \widetilde{b_{i}}\left(y_{i}\right)=\int_{0}^{1} b_{i}\left(x,\left(y_{1}, y_{2}\right)\right) d y_{j}, \quad i \neq j .
\end{aligned}
$$

Since $\int_{0}^{1} v_{i}^{\prime}\left(y_{i}\right) d y_{i}=0$, the solution is obviously obtained when

$$
\widetilde{a_{i}}\left(y_{i}\right)\left(u_{i}^{\prime}\left(y_{i}\right)+\xi_{i}\right)-\widetilde{b_{i}}\left(y_{i}\right)=k_{i}
$$

for some $k_{i}$ which is constant with respect to $y$. Thus, using that

$$
0=\int_{0}^{1} u_{i}^{\prime}\left(y_{i}\right) d y_{i}=\int_{0}^{1} \frac{k_{i}+\widetilde{b_{i}}\left(y_{i}\right)}{\widetilde{a_{i}}\left(y_{i}\right)}-\xi_{i} d y_{i}
$$

we obtain that

$$
k_{i}=\left(\int_{0}^{1}\left(\widetilde{a_{i}}\left(y_{i}\right)\right)^{-1} d y_{i}\right)^{-1}\left(\xi_{i}-\int_{0}^{1} \frac{\widetilde{b_{i}}\left(y_{i}\right)}{\widetilde{a_{i}}\left(y_{i}\right)} d y_{i}\right) .
$$

According to (8), for the solution $u \in V$ we have that

$$
f_{\xi_{i}}^{+}(x, \xi)=\int_{Y} f_{\xi_{i}}(x, y, D u+\xi) d y
$$

Thus

$$
f_{\xi_{i}}^{+}(x, \xi)=\int_{0}^{1}\left(\widetilde{a_{i}}\left(y_{i}\right)\left(u_{i}^{\prime}\left(y_{i}\right)+\xi_{i}\right)-\widetilde{b_{i}}\left(y_{i}\right)\right) d y_{i}=a_{i}^{+}(x) \xi_{i}-c_{i}^{+}(x),
$$

where

$$
\begin{aligned}
& a_{i}^{+}(x)=\left(\int_{0}^{1}\left(\widetilde{a_{i}}\left(y_{i}\right)\right)^{-1} d y_{i}\right)^{-1}, \\
& c_{i}^{+}(x)=\int_{0}^{1} \frac{\widetilde{b_{i}}\left(y_{i}\right)}{\widetilde{a_{i}}\left(y_{i}\right)} d y_{i}\left(\int_{0}^{1}\left(\widetilde{a_{i}}\left(y_{i}\right)\right)^{-1} d y_{i}\right)^{-1} .
\end{aligned}
$$

Hence,

$$
f^{+}(x, \xi)=\sum_{i=1}^{2}\left(\frac{1}{2} a_{i}^{+}(x) \xi_{i}-c_{i}^{+}(x) \xi_{i}+d_{i}^{+}(x)\right)
$$


for some function $d_{i}^{+}(x)$. In order to find $d_{i}^{+}(x)$ we first observe that $\sum_{i=1}^{2} d_{i}^{+}(x)=f^{+}(x, \xi)$ for $\xi_{i}=0$. For this value of $\xi_{i}$, we obtain from (11) that

$$
k_{i}=-\left(\int_{0}^{1}\left(\widetilde{a_{i}}\left(y_{i}\right)\right)^{-1} d y_{i}\right)^{-1}\left(\int_{0}^{1} \frac{\widetilde{b_{i}}\left(y_{i}\right)}{\widetilde{a_{i}}\left(y_{i}\right)} d y_{i}\right) .
$$

Thus (10) gives that

$$
\left(u_{i}^{\prime}\left(y_{i}\right)+\xi_{i}\right)=\frac{1}{\widetilde{a_{i}}\left(y_{i}\right)}\left(\widetilde{b_{i}}\left(y_{i}\right)-\left(\int_{0}^{1}\left(\widetilde{a_{i}}\left(y_{i}\right)\right)^{-1} d y_{i}\right)^{-1}\left(\int_{0}^{1} \frac{\widetilde{b_{i}}\left(y_{i}\right)}{\widetilde{a_{i}}\left(y_{i}\right)} d y_{i}\right)\right) .
$$

Hence,

$$
\begin{aligned}
& \frac{1}{2} \int_{0}^{1} \widetilde{a_{i}}\left(y_{i}\right)\left(u_{i}^{\prime}\left(y_{i}\right)+\xi_{i}\right)^{2} d y_{i} \\
& =\int_{0}^{1} \frac{\left(\widetilde{b_{i}}\left(y_{i}\right)-\left(\int_{0}^{1}\left(\widetilde{a_{i}}\left(y_{i}\right)\right)^{-1} d y_{i}\right)^{-1}\left(\int_{0}^{1} \frac{\widetilde{b_{i}}\left(y_{i}\right)}{\widetilde{a}_{i}\left(y_{i}\right)} d y_{i}\right)\right)^{2}}{2 \widetilde{a_{i}}\left(y_{i}\right)} d y_{i} \\
& =\frac{1}{2}\left(\int_{0}^{1} \frac{\left(\widetilde{b_{i}}\left(y_{i}\right)\right)^{2}}{\left(\widetilde{a_{i}}\left(y_{i}\right)\right)} d y_{i}-\left(\int_{0}^{1}\left(\widetilde{a_{i}}\left(y_{i}\right)\right)^{-1} d y_{i}\right)^{-1}\left(\int_{0}^{1} \frac{\widetilde{b_{i}}\left(y_{i}\right)}{\widetilde{a_{i}}\left(y_{i}\right)} d y_{i}\right)^{2}\right)
\end{aligned}
$$

and

$$
\begin{aligned}
& \int_{0}^{1} \widetilde{b_{i}}\left(y_{i}\right)\left(u_{i}^{\prime}\left(y_{i}\right)+\xi_{i}\right) d y_{i} \\
& \quad=\int_{0}^{1} \frac{\widetilde{b_{i}}\left(y_{i}\right)}{\widetilde{a_{i}}\left(y_{i}\right)}\left(\left(\widetilde{b_{i}}\left(y_{i}\right)\right)-\left(\int_{0}^{1}\left(\widetilde{a_{i}}\left(y_{i}\right)\right)^{-1} d y_{i}\right)^{-1}\left(\int_{0}^{1} \frac{\widetilde{b_{i}}\left(y_{i}\right)}{\widetilde{a_{i}}\left(y_{i}\right)} d y_{i}\right)\right) d y_{i} .
\end{aligned}
$$

Thus

$$
\begin{aligned}
d_{i}^{+}(x) & =\int_{0}^{1} \int_{0}^{1} \frac{1}{2} a_{i}(x, y)\left(u_{i}^{\prime}\left(y_{i}\right)+\xi_{i}\right)^{2}-b_{i}(x, y)\left(u_{i}^{\prime}\left(y_{i}\right)+\xi_{i}\right) d y_{j} d y_{i} \\
& =\frac{1}{2}\left(\int_{0}^{1}\left(\widetilde{a_{i}}\left(y_{i}\right)\right)^{-1} d y_{i}\right)^{-1}\left(\int_{0}^{1} \frac{\widetilde{b_{i}}\left(y_{i}\right)}{\widetilde{a_{i}}\left(y_{i}\right)} d y_{i}\right)^{2}-\frac{1}{2} \int_{0}^{1} \frac{\left(\widetilde{b_{i}}\left(y_{i}\right)\right)^{2}}{\left(\widetilde{a_{i}}\left(y_{i}\right)\right)} d y_{i} .
\end{aligned}
$$


Proof of the lower bound: The corresponding dual problem takes the form:

$$
f_{0}^{*}(x, \eta)=\min _{p \in V_{s o l}^{2}(Y)} \int_{Y} f^{*}(x, y, p+\eta) d y
$$

where $\eta \in R^{2}, V_{\text {sol }}^{2}(Y)=\left\{p \in L_{\text {sol }}^{2}(Y),\langle p\rangle=0\right\}$ and $f_{0}^{*}$ and $f^{*}$ denote the conjugate functions of $f_{0}$ and $f$ (with respect to the second and third variable, respectively), i.e.

$$
\begin{aligned}
f^{*}(x, y, \eta) & =\max \{\eta \cdot \xi-f(x, y, \xi)\} \\
& =\frac{\left(\eta_{1}+b_{1}(x, y)\right)^{2}}{2 a_{1}(x, y)}+\frac{\left(\eta_{2}+b_{2}(x, y)\right)^{2}}{2 a_{2}(x, y)} .
\end{aligned}
$$

Above, the space $L_{\text {sol }}^{2}(Y)$ is the space of soloinodal vector-fields in $\mathbf{L}^{2}(Y)$. This space is the orthogonal complement of $V_{\text {pot }}^{2}(Y)=\left\{D u: u \in W_{\text {per }}(Y)\right\}$ in $\mathbf{L}^{2}(Y)$. Hence, $\mathbf{L}^{2}(Y)=R^{2} \oplus V_{\text {sol }}^{2}(Y) \oplus V_{\text {pot }}^{2}(Y)$. Note that the minimumpoint $p=\left(p_{1}, p_{2}\right) \in V_{\text {sol }}^{2}(Y)$ is the solution of the Euler equation

$$
\int_{Y} \sum_{i=1}^{2} \frac{\left(\eta_{i}+p_{i}\left(y_{1}, y_{2}\right)+b_{i}\left(x, y_{1}, y_{2}\right)\right)}{a_{i}\left(x, y_{1}, y_{2}\right)} q_{i}\left(y_{1}, y_{2}\right) d y=0, \quad q \in V_{\text {sol }}^{2}(Y)
$$

The dual representation (16) follows by general results (see e.g. Jikov [4]). In the present situation a direct proof can be given, which we include here for completeness. Let $u=u_{\xi}+u_{0}$ where $u_{\xi}$ and $u_{0}$ are the solutions of (4) and (5), respectively. Let

$$
P=A(D u+\xi)-B .
$$

Since $\int_{Y} P \cdot D v d y=0$ for all $v \in W_{\text {per }}(Y)$ we see that $P \in L_{\text {sol }}^{2}(Y)$. Moreover, $P=\eta+\bar{p}$, where

$$
\eta=\langle P\rangle=A_{0} \xi-B_{0},
$$

and $\bar{p} \in V_{\text {sol }}^{2}(Y)$. Thus, since

$$
\frac{\left(\eta_{i}+\bar{p}_{i}+b_{i}\left(x,\left(y_{1}, y_{2}\right)\right)\right.}{a_{i}\left(x,\left(y_{1}, y_{2}\right)\right)}=\frac{\partial u}{\partial y_{i}}+\xi_{i}
$$

we obtain that $\bar{p}=p$ is the unique solution of (17), which also is the minimum-point of (16). Thus, for the function $g_{0}$ defined by

$$
g_{0}^{*}(x, \eta)=\min _{q \in V_{s o l}^{2}(Y)} \int_{Y} f^{*}(x, y, q+\eta) d y
$$


we have that

$$
\begin{aligned}
& g_{0}^{*}(x, \eta)= \sum_{i=1}^{2} \int_{0}^{1} \int_{0}^{1} \frac{\left(\eta_{i}+p_{i}+b_{i}\left(x,\left(y_{1}, y_{2}\right)\right)^{2}\right.}{2 a_{i}\left(x,\left(y_{1}, y_{2}\right)\right)} d y_{2} d y_{1} \\
&= \frac{1}{2} \sum_{i=1}^{2} \int_{0}^{1} \int_{0}^{1} a_{i}\left(x,\left(y_{1}, y_{2}\right)\right)\left(\frac{\partial u}{\partial y_{i}}+\xi_{i}\right)^{2} d y_{2} d y_{1} \\
&= \frac{1}{2} \int_{Y}(D u+\xi) \cdot A(D u+\xi) d y \\
&= \frac{1}{2} \int_{Y}\left(D u_{\xi}+\xi+D u_{0}\right) \cdot A\left(D u_{\xi}+\xi+D u_{0}\right) d y \\
&= \frac{1}{2} \int_{Y}\left(D u_{\xi}+\xi\right) \cdot A\left(D u_{\xi}+\xi\right) d y+\underbrace{\int_{Y}\left(D u_{0}\right) \cdot A\left(D u_{\xi}+\xi\right) d y}_{=0} \\
&+\frac{1}{2} \int_{Y}\left(D u_{0}\right) \cdot A\left(D u_{0}\right) d y \\
&= \frac{1}{2} \int_{Y}\left(D u_{\xi}+\xi\right) \cdot A\left(D u_{\xi}+\xi\right) d y \\
&+\frac{1}{2} \int_{Y}\left(D u_{0}\right) \cdot A\left(D u_{0}\right) d y-\int_{Y}^{B} \cdot\left(D u_{0}\right) d y \\
&= \frac{1}{2} \underbrace{}_{Y} D u_{\xi}\left(D \cdot A\left(D u_{\xi}+\xi\right) d y+\frac{1}{2} \int_{Y} \xi \cdot A\left(D u_{\xi}+\xi\right) d y-f_{0}(x, 0)\right. \\
& \int_{Y} B \cdot\left(D u_{0}\right) d y \\
&\left.\left(D u_{0}\right) d y=\frac{1}{2} \int_{Y}+\xi\right) \cdot A\left(D u_{\xi}+\xi\right) d y-f_{0}(x, 0) \\
&\left(D u_{0}(x, 0)\right.
\end{aligned}
$$

Hence, we have that

$$
g_{0}^{*}(x, \eta)=\frac{1}{2} \xi \cdot A_{0} \xi-f_{0}(x, 0),
$$

where

$$
\eta=A_{0} \xi-B_{0}
$$

Therefore,

$$
\begin{aligned}
g_{0}(x, \lambda) & =\max _{\lambda}\left\{\eta \cdot \lambda-f_{0}^{*}(x, \eta)\right\} \\
& =\max _{\xi}\left\{\left(A_{0} \xi-B_{0}\right) \cdot \lambda-\left(\frac{1}{2} \xi \cdot A_{0} \xi-f_{0}(x, 0)\right)\right\}
\end{aligned}
$$




$$
\begin{aligned}
& =-\min _{\xi}\left\{\frac{1}{2} \xi \cdot A_{0} \xi-\lambda \cdot A_{0} \xi\right\}-\lambda \cdot B_{0}+\left(f_{0}(x, 0)\right) \\
& =\frac{1}{2} \lambda \cdot A_{0} \lambda-\lambda \cdot B_{0}+\left(f_{0}(x, 0)\right) .
\end{aligned}
$$

Hence $g_{0}(x, \lambda)=f_{0}(x, \lambda)$, which proves the dual formulation. Note that above we have used that $f_{0}(x, \lambda)$ is convex (which implies that $f_{0}^{* *}(x, \cdot)=f_{0}(x, \cdot)$ by Fenchel-Moreau's theorem) and that $A_{0}$ is symmetric and positive definite, which is a standard result in homogenization theory.

Similarly as for the upper bound we prove that

$$
\left(f^{-}\right)^{*}(x, \eta)=\min _{p \in V^{*}} \int_{Y} f^{*}(x, y, p+\eta) d y
$$

where $V^{*}$ is the subspace of the space $\left\{p \in L_{\text {sol }}^{2}(Y),\langle p\rangle=0\right\}$ consisting of functions of the form

$$
p(y)=\left(p_{1}\left(y_{2}\right), p_{2}\left(y_{1}\right)\right)
$$

This will directly show that $\left(f^{-}\right)(x, \xi)$ is a lower bound to $f_{0}(x, \xi)$. Similarly as we did for the upper bound we find by using the corresponding Euler equation to (19), that the minimum is attained when

$$
\begin{aligned}
& \int_{0}^{1} \frac{\left(\eta_{1}+p_{1}\left(y_{2}\right)+b_{1}\left(x, y_{1}, y_{2}\right)\right)}{a_{i}\left(x, y_{1}, y_{2}\right)} d y_{1}=k_{1}, \\
& \int_{0}^{1} \frac{\left(\eta_{2}+p_{2}\left(y_{1}\right)+b_{2}\left(x, y_{1}, y_{2}\right)\right)}{a_{2}\left(x, y_{1}, y_{2}\right)} d y_{2}=k_{2} .
\end{aligned}
$$

Thus, since $\int_{0}^{1} p_{1}\left(y_{2}\right) d y_{2}=\int_{0}^{1} p_{2}\left(y_{1}\right) d y_{1}=0$, we obtain that

$$
\begin{aligned}
\eta_{1} & =\int_{0}^{1}\left(\eta_{1}+p_{1}\left(y_{2}\right)\right) d y_{2} \\
& =k_{1} \int_{0}^{1}\left(\int_{0}^{1} a_{1}^{-1}\left(x, y_{1}, y_{2}\right) d y_{1}\right)^{-1} d y_{2}-\int_{0}^{1} \frac{\int_{0}^{1} \frac{\left(b_{1}\left(x, y_{1}, y_{2}\right)\right)}{a_{1}\left(x, y_{1}, y_{2}\right)} d y_{1}}{\int_{0}^{1} a_{1}^{-1}\left(x, y_{1}, y_{2}\right) d y_{1}} d y_{2},
\end{aligned}
$$

and a similar expression for $\eta_{2}$. Thus for $i \neq j$,

$$
k_{i}=\left(\int_{0}^{1}\left(\int_{0}^{1} a_{i}^{-1}\left(x, y_{1}, y_{2}\right) d y_{i}\right)^{-1} d y_{j}\right)^{-1}\left(\eta_{i}+\int_{0}^{1} \frac{\int_{0}^{1} \frac{\left(b_{i}\left(x, y_{1}, y_{2}\right)\right)}{a_{i}\left(x, y_{1}, y_{2}\right)} d y_{i}}{\int_{0}^{1} a_{i}^{-1}\left(x, y_{1}, y_{2}\right) d y_{i}} d y_{j}\right) \text {. }
$$


Using the same arguments as we did for obtaining the upper bound $f^{+}$in (12), we obtain that

$$
\left(f^{-}\right)^{*}(x, \eta)=\sum_{i=1}^{2} \frac{\left(\eta_{i}+c_{i}^{-}(x)\right)^{2}}{2 a_{i}^{-}(x)}-d_{i}^{-}(x),
$$

where

$$
\begin{aligned}
a_{i}^{-}(x) & =\left(\int_{0}^{1}\left(\int_{0}^{1} a_{i}^{-1}\left(x, y_{1}, y_{2}\right) d y_{i}\right)^{-1} d y_{j}\right)^{-1}, \quad i \neq j, \\
c_{i}^{-}(x) & =\int_{0}^{1} \frac{\int_{0}^{1} \frac{\left(b_{i}\left(x, y_{1}, y_{2}\right)\right)}{a_{i}\left(x, y_{1}, y_{2}\right)} d y_{i}}{\int_{0}^{1} a_{i}^{-1}\left(x, y_{1}, y_{2}\right) d y_{i}} d y_{j}, \quad i \neq j .
\end{aligned}
$$

The function $d_{i}^{-}(x)$ is found similarly as $d_{i}^{+}(x)$ above, using that $\sum_{i=1}^{2}-d_{i}^{-}(x)=\left(f^{-}\right)^{*}(x, \eta)$ for $\eta_{i}=-c_{i}^{-}$. For this value of $\eta_{i}$, we obtain that $k_{i}=0$ and next that

$$
\eta_{i}+p_{i}\left(y_{j}\right)=-\left(\int_{0}^{1} a_{i}^{-1}\left(x, y_{1}, y_{2}\right) d y_{i}\right)^{-1} \int_{0}^{1} \frac{b_{i}\left(x, y_{1}, y_{2}\right)}{a_{i}\left(x, y_{1}, y_{2}\right)} d y_{i}, i \neq j
$$

Hence,

$$
\begin{aligned}
- & d_{i}^{-}(x) \\
= & \int_{0}^{1} \int_{0}^{1} \frac{\left(\eta_{i}+p_{i}\left(y_{j}\right)+b_{i}\left(x, y_{1}, y_{2}\right)\right)^{2}}{2 a_{i}\left(x, y_{1}, y_{2}\right)} d y_{i} d y_{j} \\
= & \int_{0}^{1} \int_{0}^{1} \frac{\left(\eta_{i}+p_{i}\left(y_{j}\right)\right)^{2}+2\left(\eta_{i}+p_{i}\left(y_{j}\right)\right) b_{i}\left(x, y_{1}, y_{2}\right)+\left(b_{i}\left(x, y_{1}, y_{2}\right)\right)^{2}}{2 a_{i}\left(x, y_{1}, y_{2}\right)} d y_{i} d y_{j} \\
= & \int_{0}^{1} \int_{0}^{1} \frac{\left(\eta_{i}+p_{i}\left(y_{j}\right)\right)^{2}}{2 a_{i}\left(x, y_{1}, y_{2}\right)} d y_{i} d y_{j}+\int_{0}^{1} \int_{0}^{1} \frac{\left(\eta_{i}+p_{i}\left(y_{j}\right)\right) b_{i}\left(x, y_{1}, y_{2}\right)}{a_{i}\left(x, y_{1}, y_{2}\right)} d y_{i} d y_{j} \\
& +\int_{0}^{1} \int_{0}^{1} \frac{\left(b_{i}\left(x, y_{1}, y_{2}\right)\right)^{2}}{2 a_{i}\left(x, y_{1}, y_{2}\right)} d y_{i} d y_{j} \\
= & \frac{1}{2} \int_{0}^{1}\left(\int_{0}^{1} a_{i}^{-1}\left(x, y_{1}, y_{2}\right) d y_{i}\right)^{-1}\left(\int_{0}^{1} \frac{b_{i}\left(x, y_{1}, y_{2}\right)}{a_{i}\left(x, y_{1}, y_{2}\right)} d y_{i}\right)^{2} d y_{j} \\
& -\int_{0}^{1}\left(\int_{0}^{1} a_{i}^{-1}\left(x, y_{1}, y_{2}\right) d y_{i}\right)^{1}\left(\int_{0}^{1} \frac{b_{i}\left(x, y_{1}, y_{2}\right)}{a_{i}\left(x, y_{1}, y_{2}\right)} d y_{i}\right)^{2} d y_{j} \\
& +\frac{1}{2} \int_{0}^{1} \int_{0}^{1} \frac{\left(b_{i}\left(x, y_{1}, y_{2}\right)\right)^{2}}{a_{i}\left(x, y_{1}, y_{2}\right)} d y_{i} d y_{j}
\end{aligned}
$$




$$
\begin{aligned}
= & \frac{1}{2} \int_{0}^{1} \int_{0}^{1} \frac{\left(b_{i}\left(x, y_{1}, y_{2}\right)\right)^{2}}{a_{i}\left(x, y_{1}, y_{2}\right)} d y_{i} d y_{j} \\
& -\frac{1}{2} \int_{0}^{1}\left(\int_{0}^{1} a_{i}^{-1}\left(x, y_{1}, y_{2}\right) d y_{i}\right)^{-1}\left(\int_{0}^{1} \frac{b_{i}\left(x, y_{1}, y_{2}\right)}{a_{i}\left(x, y_{1}, y_{2}\right)} d y_{i}\right)^{2} d y_{j} .
\end{aligned}
$$

Thus

$$
\begin{aligned}
f^{-}(x, \xi) & =\max \left\{\eta \cdot \xi-\left(f^{-}\right)^{*}(x, \eta)\right\} \\
& =\sum_{i=1}^{2} \frac{1}{2} a_{i}^{-} \xi_{i}^{2}-c_{i}^{-} \xi_{i}+d_{i}^{-}(x)
\end{aligned}
$$

Now, assume that the upper and lower bounds $f^{-}$and $f^{+}$coincide, i.e. that

$$
f^{-}(x, \xi)=f_{0}(x, \xi)=f^{+}(x, \xi) .
$$

Then

$$
\begin{aligned}
f_{0}(x, \xi) & =\min _{u \in W_{p e r}(Y)} \int_{\Omega} f(x, y, D u+\xi) d y \\
& =\min _{u \in V} \int_{\Omega} f(x, y, D u+\xi) d y .
\end{aligned}
$$

Thus, the uniqueness of the minimum-problem corresponding to $f_{0}(x, \xi)$ implies that the minimum-point $u=u_{\xi}+u_{0} \in V$. Similarly, since

$$
\left(f^{-}\right)^{*}(x, \eta)=\left(f_{0}\right)^{*}(x, \eta)
$$

the uniqueness of the minimum-problem corresponding to $\left(f_{0}\right)^{*}(x, \eta)$ implies that the minimum-point $p \in V^{*}$. Moreover, if $\eta=\eta_{\xi}+\eta_{0}$ where $\eta_{\xi}=\left\langle A\left(D u_{\xi}+\xi\right)\right\rangle, \eta_{0}=\left\langle A\left(D u_{0}\right)-B\right\rangle$, we have that $u$ and $p$ are related to each other as follows (c.f.(18)):

$$
A(D u+\xi)-B=p+\eta
$$

The solution $p$ can also be divided into two parts $p=p_{\xi}+p_{0}$, where $p_{\xi}$ and $p_{0}$ are the solutions of the dual problems corresponding to those with solutions $u_{\xi}$ and $u_{0}$, respectively. The corresponding relations are the following:

$$
\begin{gathered}
A\left(D u_{\xi}+\xi\right)=p_{\xi}+\eta_{\xi}, \\
A\left(D u_{0}\right)-B=p_{0}+\eta_{0} .
\end{gathered}
$$

Thus, since $u_{\xi}, u_{0} \in V$ and $p_{\xi}, p_{0} \in V^{*}$, the only possibility is that $a_{i}$ and $b_{i}$ is of the form $a_{i}=a_{i}^{i} a_{i}^{j}$ and $b_{i}=a_{i}^{j} f_{i}^{i}+f_{i}^{j}$. 
Conversely, if $a_{i}$ and $b_{i}$ are functions of this form, then for the solution $u \in V$ of the minimum-problem corresponding to $f_{0}^{+}(x, \xi)$ we have (c.f. (13))

$$
\begin{aligned}
& a_{i}\left(u_{i}^{\prime}\left(y_{i}\right)+\xi_{i}\right)-b_{i} \\
& =\frac{a_{i}}{\widetilde{a_{i}}\left(y_{i}\right)}\left(\widetilde{b_{i}}\left(y_{i}\right)-\left(\int_{0}^{1}\left(\widetilde{a_{i}}\left(y_{i}\right)\right)^{-1} d y_{i}\right)^{-1}\left(\int_{0}^{1} \frac{\widetilde{b_{i}}\left(y_{i}\right)}{\widetilde{a_{i}}\left(y_{i}\right)} d y_{i}\right)\right)-b_{i} \\
& =\frac{a_{i}}{a_{i}^{i} \int_{0}^{1} a_{i}^{j} d y_{j}}\left(f_{i}^{i} \int_{0}^{1} a_{i}^{j} d y_{j}+\int_{0}^{1} f_{i}^{j} d y_{j}\right) \\
& -\frac{a_{i}}{a_{i}^{i} \int_{0}^{1} a_{i}^{j} d y_{j}}\left(\int_{0}^{1}\left(\widetilde{a_{i}}\left(y_{i}\right)\right)^{-1} d y_{i}\right)^{-1} \int_{0}^{1} \frac{f_{i}^{i} \int_{0}^{1} a_{i}^{j} d y_{j}+\int_{0}^{1} f_{i}^{j} d y_{j}}{\tilde{a}_{i}\left(y_{i}\right)} d y_{i}-b_{i} \\
& =\frac{a_{i}}{a_{i}^{i} \int_{0}^{1} a_{i}^{j} d y_{j}}\left(f_{i}^{i} \int_{0}^{1} a_{i}^{j} d y_{j}-\int_{0}^{1} a_{i}^{j} d y_{j}\left(\int_{0}^{1}\left(a_{i}^{i}\right)^{-1} d y_{i}\right)^{-1}\right. \\
& \left.\times \int_{0}^{1} \frac{f_{i}^{i} \int_{0}^{1} a_{i}^{j} d y_{j}}{a_{i}^{i} \int_{0}^{1} a_{i}^{j} d y_{j}} d y_{i}\right)-b_{i} \\
& =\frac{a_{i}^{i} a_{i}^{j}}{a_{i}^{i}}\left(f_{i}^{i}-\left(\int_{0}^{1}\left(a_{i}^{i}\right)^{-1} d y_{i}\right)^{-1}\left(\int_{0}^{1} \frac{f_{i}^{i}}{a_{i}^{i}} d y_{i}\right)\right)-\left(a_{i}^{j} f_{i}^{i}+f_{i}^{j}\right) \\
& =-a_{i}^{j}\left(\int_{0}^{1}\left(a_{i}^{i}\right)^{-1} d y_{i}\right)^{-1}\left(\int_{0}^{1} \frac{f_{i}^{i}}{a_{i}^{i}} d y_{i}\right)-f_{i}^{j} .
\end{aligned}
$$

Hence $p_{i}=a_{i}\left(u_{i}^{\prime}\left(y_{i}\right)+\xi_{i}\right)-b_{i}$ is independent of $y_{i}$. Thus for any $v \in$ $W_{\text {per }}(Y)$, we have that

$$
\begin{aligned}
\int_{0}^{1}\left(a_{i}\left(u_{i}^{\prime}\left(y_{i}\right)+\xi_{i}\right)-b_{i}\right) \frac{\partial v}{\partial x_{i}} d x_{i} & =\left(a_{i}\left(u_{i}^{\prime}\left(y_{i}\right)+\xi_{i}\right)-b_{i}\right) \int_{0}^{1} \frac{\partial v}{\partial x_{i}} d x_{i} \\
& =0
\end{aligned}
$$

SO

$$
\int_{Y}\left(A(D u+\xi) D v d x_{i}=0\right.
$$

Thus $u$ is also the solution of the minimum-problem corresponding to $f_{0}(x, \xi)$. This shows that $f^{+}(x, \xi)=f_{0}(x, \xi)$. Moreover, as above we observe that

$$
A(D u+\xi)-B=p+\eta
$$

where $\eta=\langle A(D u+\xi)-B\rangle$ and $p$ is the solution of the minimum-problem corresponding to $\left(f_{0}\right)^{*}(x, \eta)$. Since $P_{i}=a_{i}\left(u_{i}^{\prime}\left(y_{i}\right)+\xi_{i}\right)-b_{i}$ is independent 
of $y_{i}$ we get that $p \in V^{*}$, and accordingly the solution of the minimumproblem corresponding to $\left(f^{-}\right)^{*}(x, \eta)$. Thus $\left(f_{0}\right)^{*}(x, \eta)=\left(f^{-}\right)^{*}(x, \eta)$, i.e. $f^{-}(x, \xi)=f_{0}(x, \xi)$, so we finally conclude that $f^{+}(x, \xi)=f^{-}(x, \xi)$.

\section{References}

[1] E. F. Beckenbach, Convexity properties of generalized mean value functions, Ann. Math. Statist., 13 (1942), 88-90.

[2] P. S. Bullen, D. S. Mitrinovic and P. M. Vasic, Means and Their Inequalities, D. Reidel Publishing Company, Dordrecht, 1988.

[3] G. H. Hardy, J. E. Littlewood and G. Polya, Inequalities, Cambridge University Press, Cambridge, 1934.

[4] V. V. Jikov(=Zhikov), S. M. Kozlov and O. A. Oleinik, Homogenization of Differential Operators and Integral Functionals, Springer-Verlag, Berlin, 1994.

[5] D. Lukkassen, Bounds and homogenization of integral functionals, Acta Sci. Math. 64 (1998), 121-141.

[6] D. Lukkassen, On estimates of the effective energy for the Poisson equation with a p-Laplacian, Russian Math. Surveys, 51 (4) (1996), 739-740.

[7] D. Lukkassen, Some sharp estimates connected to the homogenized $p$ Laplacian equation, ZAMM-Z.Angew. Math. Mech., 76 (S2) (1996), 603-604.

[8] D. Lukkassen, Formulae and bounds connected to homogenization and optimal design of partial differential operators and integral functionals, Doctoral Thesis, Department of Mathematics and Statistics, University of Troms $\varnothing$, October 1996.

[9] D. Lukkassen, L.-E. Persson and P. Wall, On some sharp bounds for the homogenized p-Poisson equation, Applicable Anal. 58 (1995), 123-135.

[10] A. Meidell, Homogenization and computational methods for calculating effective properties of some cellular solids and composite structures, Norwegian University of Science and Technology (NTNU), Doctoral thesis, Trondheim, Norway.

[11] A. Meidell and P. Wall, Homogenization and design of structures with optimal macroscopic behaviour, in : Computer Aided Optimum Design of Structures V, (Eds. : Hernández and C. A. Breddia), 393-402. Computational Mechanics Publications, Southhampton, 1997. 
[12] P. Wall, Homogenization of Reynolds equation by two-scale convergence, Research Report, No. 2, Department of Mathematics, Luleå University of Technology, 2005 (16 pages).

Dag Lukkassen and Annette Meidell

Narvik University College

P.O.B. 385 N-8505 Narvik

Norway

(E-mail : Dag.Lukkassen@hin.no)

(E-mail : am@hin.no)

\section{Peter Wall}

Department of Mathematics

Luleå University of Technology

SE-97187 Luleå

Sweden

(E-mail : wall@sm.luth.se) 


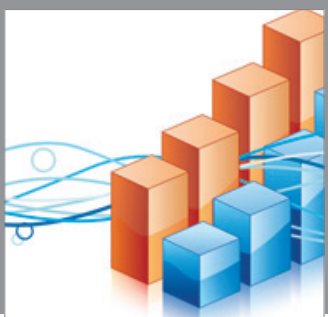

Advances in

Operations Research

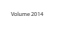

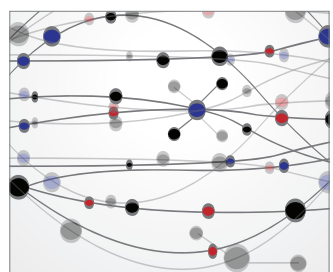

\section{The Scientific} World Journal
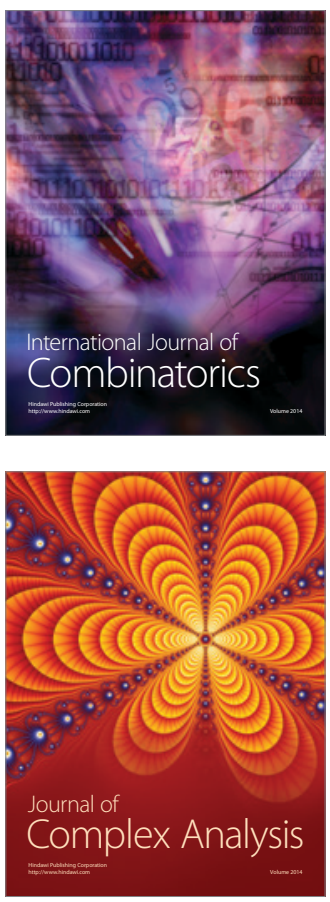

International Journal of

Mathematics and

Mathematical

Sciences
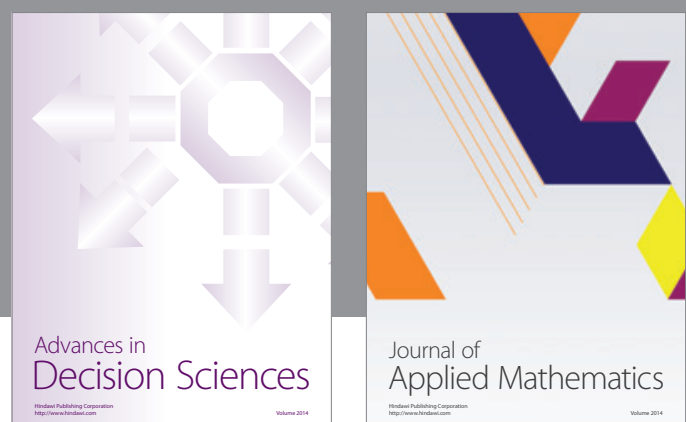

Journal of

Applied Mathematics
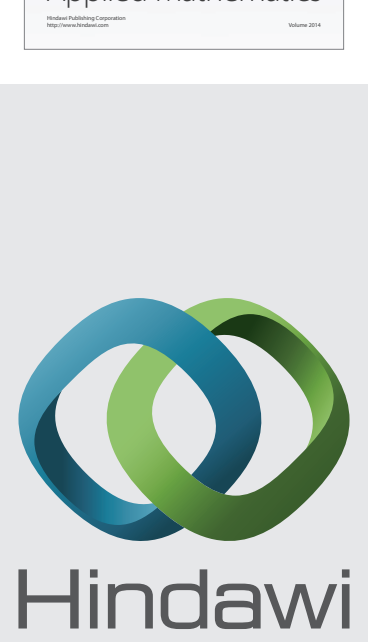

Submit your manuscripts at http://www.hindawi.com
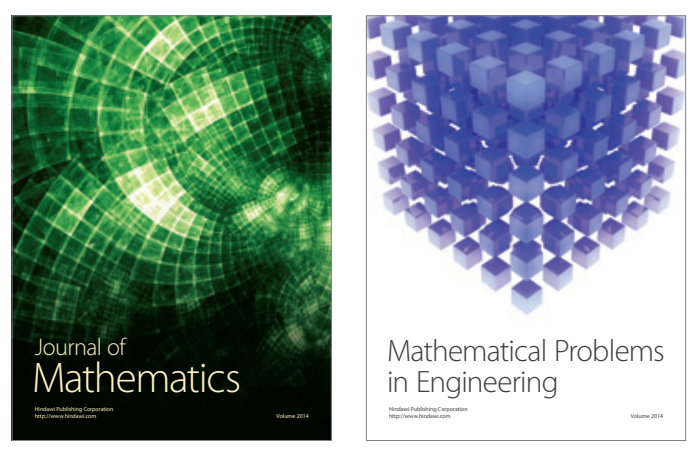

Mathematical Problems in Engineering
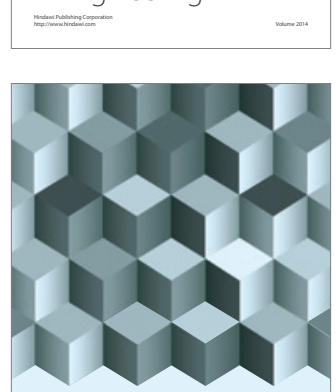

Journal of

Function Spaces
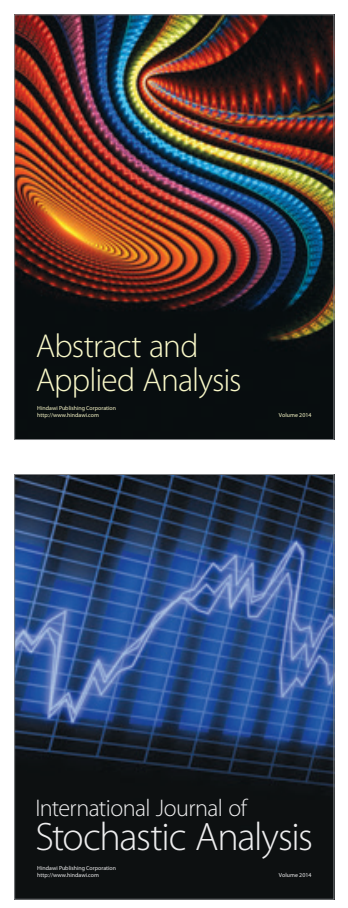

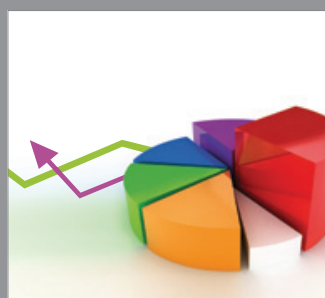

ournal of

Probability and Statistics

Promensencen
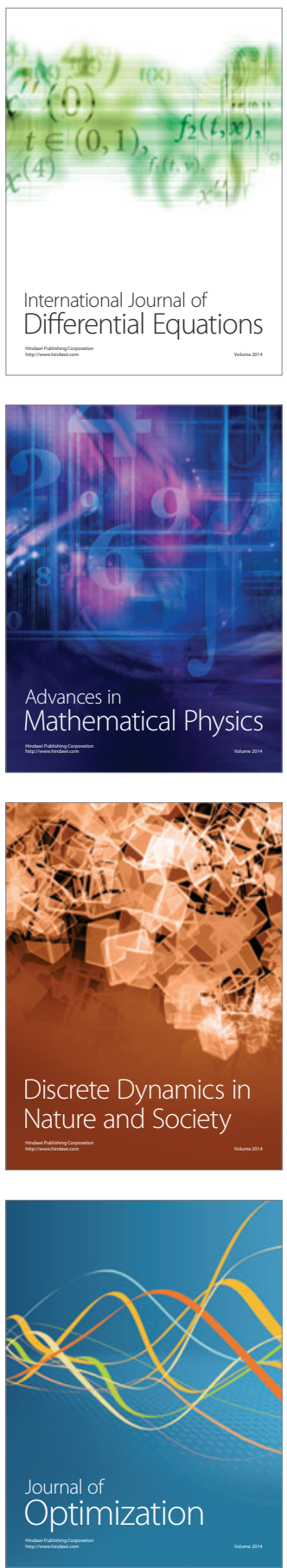